Revue internationale P.M.E.

Économie et gestion de la petite et moyenne entreprise

Revue

internationale

PME

\title{
Relation entre le profil de comportement des propriétaires-dirigeants et le stade d'évolution de leur entreprise
}

\author{
Jean Lorrain, André Belley et Charles Ramangalahy
}

Volume 7, numéro 1, 1994

URI : https://id.erudit.org/iderudit/1008368ar

DOI : https://doi.org/10.7202/1008368ar

Aller au sommaire du numéro

Éditeur(s)

Presses de l’Université du Québec

ISSN

0776-5436 (imprimé)

1918-9699 (numérique)

Découvrir la revue

Citer cet article

Jean, Belley, A. \& Ramangalahy, C. (1994). Relation entre le profil de comportement des propriétaires-dirigeants et le stade d'évolution de leur entreprise. Revue internationale P.M.E., 7(1), 9-34.

https://doi.org/10.7202/1008368ar
Résumé de l'article

Les comportements ainsi que la capacité du dirigeant à assumer certaines activités ont été identifiés parmi les facteurs de croissance, de succès d'une PME. La littérature sur les modèles de développement abonde en particulier dans ce sens. L'intérêt d'étudier leurs comportements est davantage supporté par le taux élevé de mortalité relevé. La revue que nous avons faite de la littérature et de la documentation montre, cependant, que l'étude des activités du propriétaire-dirigeant a été menée de façon partielle et sans que soit considéré le stade d'évolution des entreprises échantillonnées. Les résultats de notre recherche, menée au Québec auprès d'une cinquantaine de dirigeants de PME localisées dans la région administrative 04, révèlent des différenciations significatives par rapport aux stades relativement au comportement de délégation, au temps consacré aux activités, aux contacts externes et à la perception de certains problèmes organisationnels. 


\title{
Relation entre le profil de comportement des propriétaires-dirigeants et le stade d'évolution de leur entreprise
}

\author{
Jean LORRAIN * \\ André BELLE ** \\ Charles RAMANGALAHY*** \\ Université du Québec à Trois-Rivières
}

* Jean Lorrain est professeur en psychosociologie des PME et de l'entrepreneuriat au Département d'administration et d'économique à l'Université du Québec à TroisRivières. Il est également membre d'une équipe de recherche en management des PME qui réalise actuellement une étude internationale sur la gestion des propriétaires-dirigeants et la performance organisationnelle de leur entreprise, en collaboration avec des chercheurs de plusieurs pays. Ses articles ont paru dans diverses revues internationales. Adresse : Département d'administration et d'économique, Université du Québec à Trois-Rivières, C.P. 500, Trois-Rivières (Québec) G9A $5 \mathrm{H} 7$.

** André Belley est professeur de management, de création d'entreprises et de management des PME au Département d'administration et d'économique, en plus d'être Directeur de la maîtrise en gestion des PME. Ses activités de recherche portent sur le processus de création d'entreprises et les activités de gestion en contexte de PME. Adresse : Département d'administration et d'économique, Université du Québec à Trois-Rivières, C.P. 500, Trois-Rivières (Québec) G9A 5H7.

*** Charles Ramangalahy est étudiant au doctorat en administration à l'École des HEC de Montréal, titulaire d'une maîtrise en gestion des PME de l'Université du Québec à Trois Rivières et d'une maîtrise en sciences économiques de l'Université de Madagascar. Au cours des dernières années, il a œuvré à titre de professionnel de recherche auprès du Groupe de recherche en économie et gestion des PME. Ses intérêts de recherche présents portent sur l'internationalisation des PME et les alliances stratégiques. Adresse : Département d'administration et d'économique, Université du Québec à Trois-Rivières, C.P. 500, Trois-Rivières (Québec) G9A 5H7. 


\section{RÉSUMÉ}

Les comportements ainsi que la capacité du dirigeant à assumer certaines activités ont été identifiés parmi les facteurs de croissance, de succès d'une PME. La littérature sur les modèles de développement abonde en particulier dans ce sens. L'intérêt d'étudier leurs comportements est davantage supporté par le taux élevé de mortalité relevé. La revue que nous avons faite de la littérature et de la documentation montre, cependant, que l'étude des activités du propriétaire-dirigeant a été menée de façon partielle et sans que soit considéré le stade d'évolution des entreprises échantillonnées. Les résultats de notre recherche, menée au Québec auprès d'une cinquantaine de dirigeants de $P M E$ localisées dans la région administrative 04, révèlent des différenciations significatives par rapport aux stades relativement au comportement de délégation, au temps consacré aux activités, aux contacts externes et à la perception de certains problèmes organisationnels.

\section{ABSTRACT}

Management behaviors and managerial abilities of the owner-manager are identified as growth and success factors of SME, as demonstrated by the literature on organizational development models. The importance of studying managerial behaviors is stressed by the high failure rate experienced by that kind of organizations. Our review of the literature on the subject shows that, generally, research on the managerial activities of small business ownermanagers is incomplete and does not take into account stages of development. The results of our research on 48 SME indicate that managerial activities vary significantly with development stages, namely the delegation of authority, the time spent on these activities and on external contacts and as to the perception of organizational problems encountered.

\section{RESUMEN}

Los comportamientos y la capacidad del empresario para asumir ciertas actividades han sido identificados como factores determinantes del crecimiento y el exito de una Pequeña y Mediana Empresa (PME). La literatura sobre los modelos de desarrollo abundan de manera particular en ese sentido. La importancia de estudiar los comportamientos del empresario es confirmada por las altas tazas de mortalidad de este tipo de empresa. La revisión de la literatura muestra sin embargo, que la investigación sobre las actividades del empresario ha sido parcial y sin considerar la etapa de desarrollo de las empresas encuestadas. Los resultados de la investigación realizada en Québec con medio centenar de empresas localizadas en la "región administrativa 04" revela diferencias significativas de acuerdo a la etapa de desarrollo, con relación a la delegación de autoridad, el tiempo dedicado a las actividades de la empresa y a los contactos externos, y a la manera como el empresario percibe ciertos problemas organizacionales. 


\section{Introduction}

L'explication du succès ou de l'échec de la PME est au centre des préoccupations de recherche en entrepreneuriat (Cooper et Cascon, 1992). À priori, une telle focalisation des travaux n'a rien de particulier compte tenu de ce qui se fait dans d'autres domaines d'intérêts reliés à la gestion (Porter, 1991; Van Auken et Ireland, 1978). Cependant, elle se comprend davantage et trouve un fondement dans le taux élevé de mortalité observé chez la PME (Dodge et Robbins, 1992; Dun et Bradstreet,1981).

L'analyse rétrospective des recherches (Dussault, 1987) semble montrer que la tendance a été de rechercher l'explication au niveau du profil psychologique du dirigeant et des caractéristiques organisationnelles des entreprises. Récemment, Gartner (1988) a soulevé tout simplement l'échec ou du moins l'inadéquation de l'approche basée sur les traits psychologiques. D'un autre côté, les recherches sur les caractéristiques organisationnelles ont plutôt conduit à l'identification de plusieurs typologies de PME (Filley et Aldag, 1978). Cela a incité de plus en plus de chercheurs à recommander la réorientation des travaux sur les comportements du dirigeant ou de l'entrepreneur (Sandberg et Hofer, 1987; Stevenson et Jarillo, 1990). Dans une étude sur les facteurs de succès, Ibrahim et Godwin (1986) ont conclu que le comportement de l'entrepreneur est un des deux principaux facteurs du succès de la PME. Pour leur part, Lorrain et Dussault (1988) ont relevé, dans une étude longitudinale, que le profil psychologique ne permettait pas de différencier les PME qui ont survécu. Le caractère récent de ce revirement est quelque peu surprenant en raison de ce qui se fait depuis longtemps, par exemple, en leadership (Aldrich et Zimmer 1986), en management (Mintzberg, 1984; Kotter, 1982; Stewart, 1986), ou avec les thèses généralement développées dans les modèles de croissance (Greiner, 1972; Toulouse, 1982; Kroeger, 1974; Kazajian, 1984).

Les préoccupations de la présente recherche s'inscrivent dans l'approche comportementale suggérée par plusieurs chercheurs. Notre revue de la documentation nous a permis de constater que non seulement peu de chercheurs se sont intéressés aux comportements du dirigeant de la PME (Castaldi, 1986), mais que plus de la majorité de ceux qui l'ont fait n'ont pas tenu compte de leur différenciation en relation avec l'évolution de l'entreprise (Vozikis et Mescon, 1985). D'une façon plus spécifique, dans cette recherche, nous nous intéressons à leurs activités de gestion, leurs relations externes ainsi qu'à leurs perceptions des problèmes organisationnels. Notre hypothèse de recherche est que le profil de comportement des dirigeants se différencie en relation avec le stade de développement de leur entreprise. Nous mesurons successivement le profil de comportement par l'étendue du champ d'activité de gestion, le niveau d'implication dans ces activités et le temps consacré. 
Nous présentons d'abord nos travaux de synthèse de la littérature et notre cadre conceptuel, pour ensuite exposer la méthodologie utilisée ainsi que l'analyse des résultats obtenus. Nous soulevons dans la conclusion les implications et les limites de notre recherche.

\section{Revue de la littérature}

\subsection{Taxonomies d'activités du dirigeant}

Un fait marquant de notre revue de la littérature a été de relever l'absence de cadre d'analyse (taxonomie) sur les activités d'un dirigeant de PME. Cela est surprenant vu qu'un nombre relativement important de chercheurs ont soulevé le rôle et l'impact déterminant des comportements du dirigeant sur l'organisation et les performances d'une PME (Castaldi, 1986; Dollinger, 1985; Toulouse, 1982; Steinmetz, 1969; Greiner, 1972; Kroeger, 1974; Kazajian, 1984; Churchill et Lewis, 1983).

Contrairement à celui d'une grande entreprise, le dirigeant d'une PME (au sens de propriétaire-dirigeant) présente la particularité d'être à la fois un gestionnaire, un administrateur et un exécutant. Cela est en particulier vrai au stade de démarrage. À ce titre, d'ailleurs, plusieurs auteurs l'identifient à «l'homme à tout faire» (Churchill et Lewis, 1983; Steinmetz, 1969; Broom et al., 1983). Il est impliqué directement dans les opérations (Choran, 1969; Castaldi, 1986), la gestion (Gable et Topol, 1987; Robinson et al., 1986; Sexton et Van Auken, 1982) mais aussi dans des activités de nature administrative (Deeks, 1976).

En référence implicite aux réponses qu'apportent les écoles de pensée en management, Hales (1986) note qu'il peut paraitre naïf ou tautologique de se poser la question: «Que fait un dirigeant?» Les travaux empiriques dans ce domaine ont presque exclusivement porté sur les dirigeants de grandes entreprises. Dans une des plus récentes revues de la littérature sur le sujet, Hales (1986) a relevé six taxonomies, soit celles de Hemphill, (1959), Mintzberg (1984), Pheysey (1972), Sayles (1964), Kotter (1982) et Stewart (1982). Nous avons complété cette synthèse par celles de Tornow et Pinto (1976), Luthans et Lockwood (1984) et de Hales (1986). Bien que visant à répondre à une même question («Que fait un dirigeant?»), les modèles proposés se différencient à plusieurs égards: les catégories de cadres ciblés, l'optique de modélisation, la classification et la définition des activités.

La constatation de la pertinence limitée de ces taxonomies au contexte de la PME nous a amenés à procéder à une synthèse, dont nous avons complété et analysé le contenu par rapport à la littérature pertinente (Castaldi, 1986; Churchill et Lewis, 1983 ; Dollinger, 1985; Paolillo, 1984; Robinson et al. 
1986 ; Mc Evoy, 1984 ; Deeks, 1976). Pour résoudre la forte redondance et l'élasticité relevées dans la définition des activités (Hales, 1986) ${ }^{1}$, nous nous sommes référés à l'approche classique du management (Laflamme, 1978; Newman et al., 1982 ou Haimann et al., 1982, dans : Miner et Luschinger, 1985).

La reclassification des activités obtenues de la synthèse a permis de dégager un modèle selon lequel un dirigeant assume trois groupes d'activités (figure 1).

FIGURE 1

Modèle d'analyse des activités du dirigeant

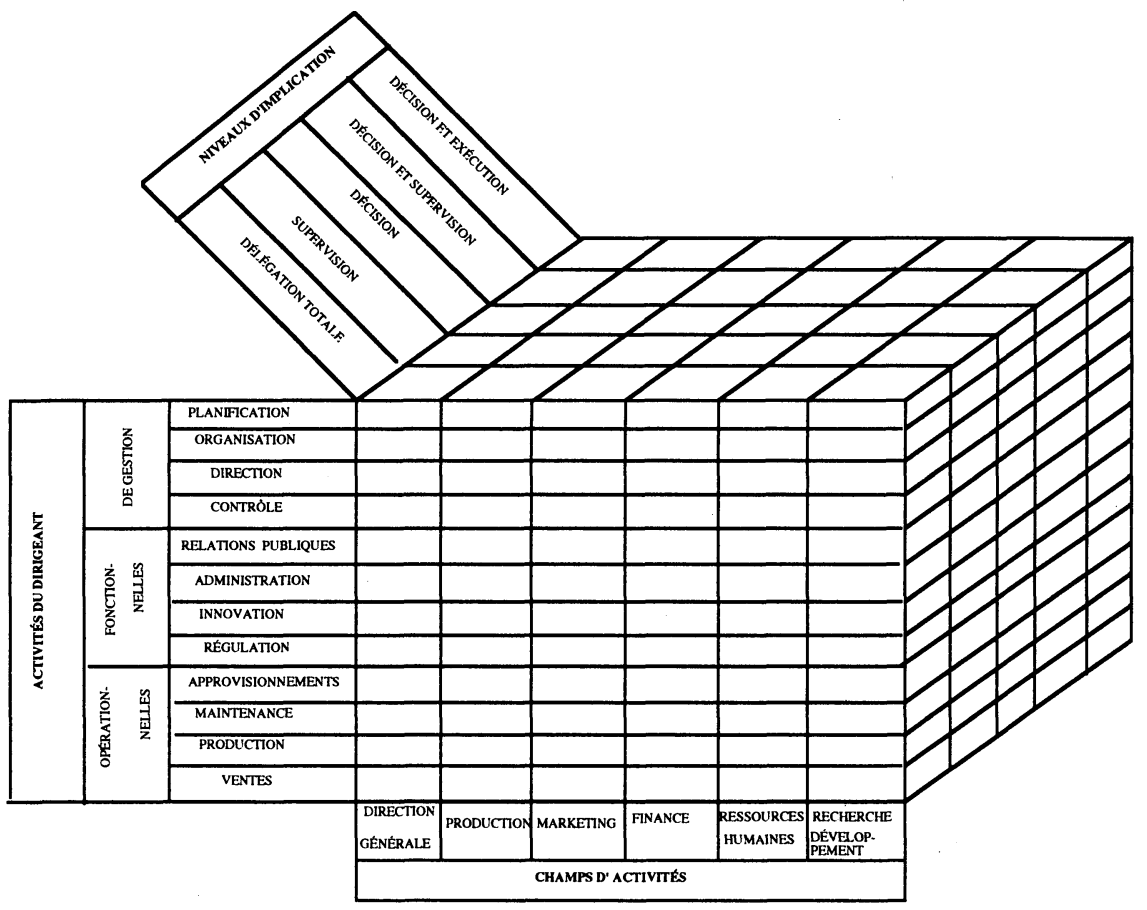

1. Les activités de programmation et de budgétisation sont généralement associées à la planification (cf. Laflamme, 1978; Miner et Luchsinger, 1985). Or, dans les modèles de Tornow et Pinto (1976) et de Hemphill (1967), elles sont rattachées à la supervision et au contrôle des performances. Certaines activités sont définies à plus de deux ou trois niveaux. Hemphill (1967) inclut notamment dans les responsabilités opérationnelles : le développement de nouvelles activités (innovation), l'anticipation des nouveaux besoins ou des changements au niveau de la demande (planification stratégique), l'assistance au personnel de vente dans la réalisation des contrats importants ou dans le recouvrement des comptes (ventes) et le maintien de relations étroites avec les clients (relations publiques). 
La première dimension réfère à des activités de gestion, dans lesquelles le dirigeant prévoit l'avenir de son entreprise (la planification); il élabore la structure d'organisation (l'organisation); mobilise les ressources humaines (la direction); et effectue le suivi des réalisations et procède aux ajustements (contrôle). Dans la deuxième dimension, qui est de nature fonctionnelle, le dirigeant assume des activités de nature administrative (l'administration); il développe des relations externes et assure la promotion des intérêts de son entreprise (les relations publiques); il initie et implante des changements (l'innovation); et il résout des problèmes ou des crises liés aux activités quotidiennes (la régulation). Enfin, dans la troisième dimension, de nature opérationnelle, le dirigeant (exécutant) assure l'approvisionnement en matières premières et en marchandises (l'approvisionnement); il maintient en état les équipements disponibles (la maintenance); il produit ou fournit les produits/ services (la production); et il assure la vente des produits/services (la vente).

Théoriquement, ce modèle présente l'avantage d'intégrer les apports de l'école dite «classique» du management et ceux de l'école dite de «l'activité du cadre», mais aussi d'intégrer et de dissocier les activités selon leur nature ainsi que de permettre une analyse par fonction.

\subsection{Développement de la PME et activités du dirigeant}

Une des approches dominantes dans l'analyse du développement de la PME a consisté dans l'élaboration de modèles de métamorphose, dont la particularité réside dans l'accent mis sur la nature des changements organisationnels (Raymond et Lorrain, 1992 ; d'Amboise et Muldowney, 1988). La thèse soutenue par les tenants de cette approche consiste en un schéma postulant l'existence d'une séquence de stades normaux d'évolution de la petite entreprise (Stanworth et Curran, 1976).

Plusieurs modèles ont été élaborés pour décrire l'évolution d'une petite entreprise: Thain (1969), Steinmetz (1969), Greiner (1972), Kroeger (1974), Basire (1976), Naumes (1978), Cooper (1979), Scott (1979), Toulouse (1982), Beckman et al. (1982), Quinn et Cameron (1983), Broom et al. (1983), Churchill et Lewis (1983), Kajarian, 1984, et Smith et al. (1985). Une des thèses avancées pour expliquer l'échec ou la réussite s'articule autour du propriétaire-dirigeant et se réfère notamment à ses capacités d'assumer certaines activités, à relever des défis ou à résoudre des problèmes qui sont caractéristiques du stade d'évolution de son entreprise (Kazajian, 1984; Churchill et Lewis, 1983; Toulouse, 1982; Greiner, 1982; Kroeger, 1974).

Le nombre de stades de ces modèles varie de 3 (Cooper, 1979) à 11 (Parks, 1977) et, plus généralement, il s'élève à 5 ou 6 . Toutefois, dans la plupart de ces 
modèles, certains stades dépassent le cadre général de définition d'une PME, soit du point de vue de la taille, de la formalisation ou de la standardisation (Susbauer, 1979; Churchill, 1983, Stegall et al., 1976; Steinmetz, 1979; Cooper, 1979). L'exclusion de ces stades de notre analyse nous a amenés à des modèles comportant trois stades. Par la suite, nous avons comparé et reclassifié les modèles en fonction de deux critères qui sont utilisés plus ou moins implicitement par la majorité des auteurs pour différencier les stades de leur modèle: les préoccupations organisationnelles et l'état de profitabilité de l'entreprise. Cela a permis d'élaborer un modèle de synthèse à trois stades: démarrage, rentabilisation et réussite (figure 2).

FIGURE 2

\section{Modèle d'analyse de la croissance d'une PME}

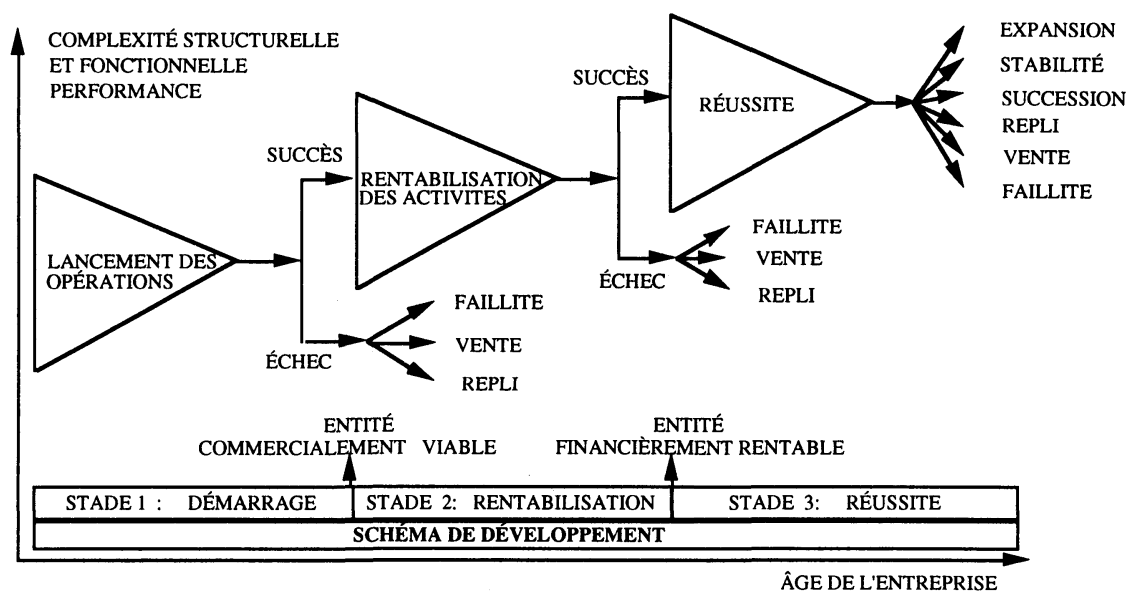

Le stade de démarrage est marqué par le lancement des activités commerciales de production et de vente. Stratégiquement, il s'agit de pénétrer le marché. Le dirigeant occupe une place centrale dans la prise de décision, la réalisation des activités et le contrôle. La structure d'organisation est simple, informelle et centralisée. Les systèmes formels sont minimes, rudimentaires. Les problèmes auxquels l'entreprise doit faire face sont liés aux opérations et son niveau de rentabilité est nul ou faible. Au terme de ce stade, l'entreprise est devenue une entité commercialement viable et implantée sur le marché. Les préoccupations fondamentales au stade de rentabilisation consistent à assurer l'efficacité des activités, à équilibrer les dépenses et les revenus. L'entreprise a pu grandir en taille après l'embauche de nouveaux responsables, à qui le dirigeant délègue certaines responsabilités opérationnelles. Le dirigeant conserve une place centrale dans la prise des décisions importantes, tout en entretenant un 
contact direct avec les activités. Tout en évoluant vers une situation de rentabilité, l'entreprise doit trouver des solutions à des problèmes de liquidité, alors que le dirigeant est soumis à des pressions croissantes à déléguer. Au stade de réussite, l'entreprise est devenue une entité économiquement rentable et socialement reconnue. L'entreprise s'est souvent agrandie, et elle est structurée par fonction. Le dirigeant concentre ses activités sur la gestion et la prise de décisions stratégiques. Il n'entretient un contact direct qu'avec les activités importantes. À la délégation fonctionnelle et la planification opérationnelle s'ajoute la planification stratégique. Quel que soit le stade atteint par une entreprise, il semble que celle-ci ne soit jamais à l'abri d'un échec absolu (faillite) ou relatif (repli stratégique ou forcé), et que le dirigeant peut à tout moment interrompre la poussée de son entreprise, procéder à sa vente ou se faire remplacer à la direction (Churchill et Lewis, 1983; Vozikis et Glueck, 1980; Toulouse, 1982).

Ces modèles décrivent de façon minimale les activités du dirigeant. Au démarrage, un consensus assez net se dégage quant au fait que c'est aux activités de production et de vente que les dirigeants consacrent essentiellement leur temps : créer un produit et un marché, rechercher une clientèle, produire, vendre et distribuer en quantité suffisante, constituer une niche (Churchill, 1983, Churchilll et Lewis, 1983; Toulouse, 1982 ; Cooper, 1979; Steinmetz, 1969; Greiner, 1972). Le dirigeant est impliqué dans toutes les fonctions de l'entreprise, mais consacre essentiellement son temps à la production et à la vente (Toulouse, 1982; Steinmetz, 1969; Greiner, 1972). Le stade de rentabilisation est considéré par plusieurs comme un stade de mise en place des mécanismes de gestion (Greiner, 1972; Beckman et al. 1982; Steinmetz, 1969; Toulouse, 1982). Beckman et al. (1982) ainsi que Kajazian (1984) soulignent que le dirigeant consacre, à ce stade, encore une part prépondérante de son temps aux opérations. Au stade de réussite, les activités du dirigeant tendent à se concentrer sur la gestion: prise de décision stratégique, planification opérationnelle et stratégique, supervision et coordination fonctionnelles des cadres à qui les responsabilités opérationnelles sont maintenant déléguées (Cooper, 1969; Beckman et al., 1982; Toulouse 1982; Churchill et Lewis 1983).

\section{Méthodologie}

\subsection{Procédure d'enquête}

Au total, deux cents questionnaires ont été distribués à des dirigeants d'entreprises de la région 04 du Québec (Mauricie et Bois-Francs) par voie postale, à partir d'une banque de données fournies par le Direction régionale du ministère de l'Industrie, du Commerce et de la Technologie. Le questionnaire comprend six parties qui portent sur l'analyse des caractéristiques des dirigeants, de leurs 
réseaux de contact, et des caractéristiques générales de leur entreprise et de leurs activités.

Avant l'enquête, un prétest a été réalisé auprès d'une dizaine de dirigeants en vue d'améliorer la clarté et la structure du questionnaire, qui prenait en moyenne une heure et demie à remplir. Des soixante questionnaires retournés, nous en avons retenu quarante-huit pour le traitement, ce qui correspond à un taux de réponse de $24 \%$, ce qui est acceptable.

\subsection{Instruments de mesure}

La grille d'analyse des activités comporte 85 variables regroupées en trois dimensions : les activités de gestion (56 variables), les activités fonctionnelles (21) et les activités opérationnelles (8). L'implication des dirigeants dans ces activités a été mesurée sur une échelle de type Likert à cinq points alors que le temps qu'ils y consacrent a été mesuré en pourcentage du temps total. La grille d'analyse de contacts externes a été développée à partir des travaux de Dollinger (1985). Elle comporte 13 variables (type de contact externe). Leur importance et fréquence relatives ont été mesurées sur une échelle de type Likert à cinq points. La grille d'analyse des problèmes organisationnels a été élaborée à partir des travaux de Churchill et Lewis (1983); elle comporte 19 variables regroupées par rapport aux fonctions de production, marketing, gestion, ressources humaines et finances. Pour mesurer le stade de développement des entreprises, nous avons utilisé la grille tridimensionnelle développée par Lorrain et Raymond (1992) employant la méthode des scénarios et comporte 16 variables. Leur modèle présente l'avantage de permettre de tenir compte, simultanément, de l'évolution de chaque entreprise sur le plan structurel, fonctionnel et de la performance.

\subsection{Traitement et analyse des données}

Avec la taille de notre échantillon, nous avons utilisé le logiciel SPSS, version $\mathrm{X}$ de Nie et al. (1975) pour traiter les données. Dans une première étape, nous avons recouru aux statistiques descriptives pour établir le profil sociodémographique des dirigeants et les caractéristiques globales de leur entreprise. Pour classer les entreprises par stade, nous avons procédé de la manière suivante. Avec une moyenne pour les trois dimensions inférieure ou égale à 1,70 , une entreprise est classée au stade de démarrage. Entre 1,71 et 2,40, elle est classée au stade de rentabilisation. À partir de 2,41, elle est classée au stade de réussite. Pour le reste du traitement, nous avons recouru à des outils plus spécifiques. Pour vérifier l'existence de corrélation par rapport aux stades des variables étudiées, nous avons utilisé le test de Kendall (Tau) vu que la variable indépen- 
dante (stade de développement) est non dichotomique. Pour vérifier l'existence de différenciation entre les stades, nous avons utilisé le test non paramétrique de Kruskal-Wallis $(F)$ quand la variable dépendante était de type nominal ou ordinal (niveau d'implication dans les activités, importance et fréquence des contacts, importance des problèmes organisationnels), et le test Oneway (Chi-deux) quand la variable dépendante était de type métrique (nombre d'activités, pourcentage de temps, nombre de contacts).

\section{4. Échantillon d'étude}

Les répondants sont majoritairement des hommes ( $85 \%)$, dirigeant des entreprises manufacturières (77\%). Âgés en moyenne de 45 ans, ils ont plutôt un bon bagage scolaire (14 années de scolarité en moyenne); plus de $60 \%$ ont au moins suivi des études collégiales et $46 \%$ des études universitaires. Ils sont en majorité $(56 \%)$ fondateurs de leur entreprise.

Plutôt âgées, avec une moyenne d'âge de 17 ans (depuis leur fondation), leurs entreprises présentent en moyenne, du point de vue de la taille, un effectif de 19 employés, 2,5 millions de dollars de chiffre d'affaires et 1,25 million d'actif. Douze d'entre elles ( $25 \%$ ) se trouvaient au stade de démarrage, vingtquatre $(50 \%)$ au stade de rentabilisation et douze $(25 \%)$, au stade de réussite.

\section{Présentation des résultats}

\subsection{Profil de comportement dans les activités}

Au préalable, nous avons tenu à vérifier si les dirigeants opéraient à l'intérieur d'un champ d'activités relativement homogène de façon à asseoir le pertinence des analyses que nous ferions sur leur niveau d'implication et la répartition de leur temps. En effet, nos analyses et résultats perdraient leur sens dans le cas où ce champ d'activité serait hétérogène par rapport aux stades.

Ce premier traitement a été réalisé à même les données sur le niveau d'implication des dirigeants dans les activités. Par définition, une activité est assumée par les dirigeants à condition qu'ils soient impliqués, soit dans la prise de décision, dans la supervision ou dans l'exécution (tableau 1, première colonne). Les résultats du test de Kendall font apparaître un élargissement significatif de l'étendue des activités assumées par rapport aux stades, en ce qui concerne les trois groupes d'activité à 0,05 , et de façon spécifique pour les activités de régulation et d'approvisionnement (à 0,01$)$, de planification, de direction et d'innovation (à 0,05$)$. Ces résultats signifient donc que d'un stade à l'autre, les dirigeants assument un nombre croissant de tâches associées à ces 
TABleAu 1

Étendue $^{1}$ et niveau d'implication ${ }^{2}$ dans les activités selon les stades

\begin{tabular}{|c|c|c|c|c|c|c|c|c|c|c|c|c|c|}
\hline \multirow{2}{*}{$\begin{array}{l}\text { DIMENSIONS } \\
\text { D'ACTIVITE }\end{array}$} & \multicolumn{6}{|c|}{ ETENDUE DES ACTIVTTES } & \multicolumn{7}{|c|}{ NIVEAU D'IMPLICATION } \\
\hline & $\overline{\mathbf{x}} \mathbf{1}$ & $\overline{\mathbf{x} 2}$ & $\overline{\mathbf{x 3}}$ & $\begin{array}{l}\text { Tau de } \\
\text { Kendall }\end{array}$ & $\begin{array}{l}\text { F de } \\
\text { Oneway }\end{array}$ & $\bar{x} 1$ & $\sigma 1$ & $\overline{x_{2}}$ & $\sigma 2$ & $\overline{\mathbf{x} 3}$ & $\sigma 3$ & $\begin{array}{l}\text { Tau de } \\
\text { Kendall }\end{array}$ & $\mathbf{K} \cdot \mathbf{W}$ \\
\hline $\begin{array}{l}\text { Activités de gestion } \\
\text { - Planification } \\
\text { - Organisation } \\
\text { - Direction } \\
\text { - Contrôle }\end{array}$ & $\begin{array}{l}83 \\
83 \\
74 \\
89 \\
90\end{array}$ & $\begin{array}{l}84 \\
86 \\
76 \\
89 \\
81\end{array}$ & $\begin{array}{r}95 \\
94 \\
96 \\
100 \\
95\end{array}$ & $\begin{array}{l}0,27^{*} \\
0,27^{*} \\
0,20 \\
0,23^{*} \\
0,17\end{array}$ & $\begin{array}{l}2,57 \\
2,08 \\
2,28 \\
0,94 \\
2,32\end{array}$ & $\begin{array}{l}1,44 \\
1,44 \\
2,30 \\
1,53 \\
1,46\end{array}$ & $\begin{array}{l}0,67 \\
0,70 \\
0,61 \\
1,11 \\
0,64\end{array}$ & $\begin{array}{l}2,19 \\
2,18 \\
2,16 \\
2,08 \\
2,41\end{array}$ & $\begin{array}{l}0,78 \\
0,75 \\
2,53 \\
1,02 \\
1,05\end{array}$ & $\begin{array}{l}2,71 \\
2,73 \\
2,53 \\
2,25 \\
2,89\end{array}$ & $\begin{array}{l}1,00 \\
1,07 \\
0,97 \\
1,09 \\
1,17\end{array}$ & $\begin{array}{l}0,42 * * * \\
0,41 * * * \\
0,40 * * * \\
0,27 * \\
0,39 * * *\end{array}$ & $\begin{array}{l}13,07 * * \\
12,57 * * \\
11,58 * * \\
5,90 \\
10,88 * *\end{array}$ \\
\hline $\begin{array}{l}\text { Activites } \\
\text { fonctionnelles } \\
\text { - Administration } \\
\text { - Relations publiques } \\
\text { - Innovation } \\
\text { - Regulation }\end{array}$ & $\begin{array}{l}83 \\
93 \\
90 \\
70 \\
79\end{array}$ & $\begin{array}{l}86 \\
95 \\
88 \\
80 \\
83\end{array}$ & $\begin{array}{l}94 \\
98 \\
93 \\
88 \\
98\end{array}$ & $\begin{array}{l}0,26 * \\
0,15 \\
0,01 \\
0,21 * \\
0,37^{* *}\end{array}$ & $\begin{array}{l}1,69 \\
0,74 \\
0,57 \\
1,16 \\
2,74\end{array}$ & $\begin{array}{l}1,49 \\
1,76 \\
1,15 \\
1,40 \\
1,70\end{array}$ & $\begin{array}{l}0,61 \\
0,90 \\
0,44 \\
0,69 \\
0,90\end{array}$ & $\begin{array}{l}2,21 \\
2,42 \\
1,86 \\
2,29 \\
2,30\end{array}$ & $\begin{array}{l}0,88 \\
1,20 \\
0,97 \\
1,00 \\
1,16\end{array}$ & $\begin{array}{l}2,96 \\
3,62 \\
2,45 \\
2,74 \\
3,13\end{array}$ & $\begin{array}{l}0,77 \\
0,99 \\
0,82 \\
0,88 \\
1,06\end{array}$ & $\begin{array}{l}0,45 * * * \\
0,42 * * * \\
0,48 * * * \\
0,40 * * * \\
0,39 * * *\end{array}$ & $\begin{array}{c}14,55^{* * *} \\
13,44^{* *} \\
5,79 * * * \\
11,83^{* *} \\
10,10 * *\end{array}$ \\
\hline $\begin{array}{l}\text { Activités } \\
\text { opérationnelles } \\
\text { - Ventes } \\
\text { - Approvisionnement } \\
\text { - Maintenance } \\
\text { - Production }\end{array}$ & $\begin{array}{l}75 \\
96 \\
79 \\
59 \\
67\end{array}$ & $\begin{array}{r}93 \\
100 \\
98 \\
88 \\
86\end{array}$ & $\begin{array}{r}98 \\
100 \\
00 \\
75 \\
78\end{array}$ & $\begin{array}{l}0,29 * \\
0,19 \\
0,37 * * \\
0,17 \\
0,16\end{array}$ & $\begin{array}{l}3,55^{*} \\
1,53 \\
5,27^{* *} \\
2,12 \\
1,03\end{array}$ & $\begin{array}{l}2,03 \\
1,79 \\
1,91 \\
3,19 \\
2,15\end{array}$ & $\begin{array}{l}1,35 \\
1,53 \\
1,39 \\
1,65 \\
1,58\end{array}$ & $\begin{array}{l}2,88 \\
2,21 \\
2,50 \\
3,83 \\
3,38\end{array}$ & $\begin{array}{l}1,10 \\
1,22 \\
1,45 \\
1,49 \\
1,56\end{array}$ & $\begin{array}{l}3,90 \\
3,29 \\
3,96 \\
4,61 \\
4,40\end{array}$ & $\begin{array}{l}0,86 \\
1,03 \\
0,96 \\
0,70 \\
1,08\end{array}$ & $\begin{array}{l}0,42 * * * \\
0,43 * * * \\
0,41 * * * \\
0,27^{*} \\
0,44 * * *\end{array}$ & $\begin{array}{c}12,04 * * \\
11,44^{*} * \\
1,54 * * \\
3,91 \\
10,35 * *\end{array}$ \\
\hline
\end{tabular}

$* \mathrm{p}<0,05 \quad * * \mathrm{p}<0,01 \quad * * * \mathrm{p}<0,001$

1. Mesurée en $\%$ du nombre d'activités assumées par groupe d'activités.

2. Échelle allant de $1=$ Le dirigeant prend les décisions et assume lui-même l'exécution de l'activité à $5=$ Il délègue les décisions,

la supervision et l'exécution. 
activités. Cependant, le test de F-Oneway révèle que des différences significatives ne prévalent entre les stades que pour les activités opérationnelles globalement $(a ̀$ 0,05) et en particulier, l'activité d'approvisionnement (à 0,01$)$. Aussi, malgré la tendance qui ressort du test de Kendall, peut-on soutenir en définitive que les dirigeants opèrent à l'intérieur d'un champ d'activité relativement homogène, sachant que l'étendue des activités qu'ils assument est comparable. Ce dernier résultat est plutôt intéressant dans la mesure où il nous permet de comparer de façon saine l'implication et le temps consacré aux activités.

Par la suite, nous avons analysé l'implication des dirigeants dans ces activités, dans le but d'appréhender leur comportement relativement à la délégation (tableau 1, deuxième colonne). L'ensemble des résultats révèle une très nette tendance à la délégation. En effet, les résultats globaux (par groupe d'activités) et détaillés (par activité) du test de Kendall sont significatifs à 0,001 , sauf pour les activités de direction et de maintenance (à 0,05$)$. L'observation des moyennes pour ces deux activités permet d'expliquer l'absence de différenciation. En effet, il y ressort que l'activité de direction est en fait une parmi les moins déléguées à tous les stades, alors que l'activité de maintenance est quasi totalement déléguée dès le stade de démarrage. Le test de Kruskal-Wallis fait ressortir que des différences significatives prévalent entre les stades à au moins 0,01 , à l'exception des activités de direction et d'approvisionnement.

Dans une troisième étape, nous avons analysé le temps consacré aux activités (tableau 2). Les données relatives aux moyennes montrent que les dirigeants des entreprises aux stades de démarrage et de rentabilisation consacrent globalement le plus de temps aux activités opérationnelles et c'est l'inverse, pour les activités de gestion. Par contre, ceux des entreprises au stade de réussite consacrent le plus de temps aux activités fonctionnelles et le moins de temps aux activités opérationnelles. Les résultats par activité montrent qu'aux stades de démarrage et de rentabilisation, les dirigeants consacrent le plus de temps à la vente et le moins de temps à la maintenance. Au stade de réussite, ce sont les activités d'administration et de production qui priment. En ce qui concerne en particulier les activités de gestion, on relève au stade de démarrage que les dirigeants allouent plus de temps à la direction, alors qu'aux stades de rentabilisation et de réussite, c'est à la planification qu'ils consacrent le plus de leur temps. Des activités fonctionnelles, c'est à l'administration que les dirigeants allouent le plus de temps, tandis que parmi les activités opérationnelles, c'est la vente qui prime à tous les stades.

Les résultats globaux du test de Kendall montrent que les dirigeants consacrent significativement de plus en plus de temps aux activités de gestion (à $0,001$ ) et fonctionnelles (à 0,01$)$ et, inversement, de moins en moins de temps aux opérations $(0,001)$. Les résultats détaillés révèlent une diminution du temps consacré à cinq activités, mais ils ne sont significatifs que dans le cas de celles 
TABLEAU 2

Temps consacré aux activités selon les stades (en \% du temps total)

\begin{tabular}{|c|c|c|c|c|c|c|c|c|}
\hline $\begin{array}{l}\text { DIMENSIONS } \\
\text { D'ACTIVITÉS }\end{array}$ & $\overline{\mathbf{x}} \mathbf{1}$ & $\sigma 1$ & $\overline{\mathbf{x} 2}$ & $\sigma 2$ & $\overline{\mathbf{x} 3}$ & $\sigma 3$ & $\begin{array}{l}\text { Tau de } \\
\text { Kendall }\end{array}$ & $\begin{array}{l}\text { F de } \\
\text { Oneway }\end{array}$ \\
\hline $\begin{array}{l}\text { Activités de gestion } \\
\text { - Planification } \\
\text { - Organisation } \\
\text { - Direction } \\
\text { - Contrôle }\end{array}$ & $\begin{array}{r}16,79 \\
4,28 \\
3,80 \\
4,90 \\
3,80\end{array}$ & $\begin{array}{r}11,95 \\
3,40 \\
3,91 \\
5,83 \\
3,58\end{array}$ & $\begin{array}{r}25,47 \\
8,64 \\
5,99 \\
5,76 \\
5,07\end{array}$ & $\begin{array}{r}15,51 \\
7,74 \\
4,27 \\
4,47 \\
3,40\end{array}$ & $\begin{array}{r}39,47 \\
12,60 \\
6,93 \\
12,50 \\
7,43\end{array}$ & $\begin{array}{r}18,95 \\
9,06 \\
3,03 \\
6,23 \\
4,53\end{array}$ & $\begin{array}{l}0,35 * * * \\
0,30 * * \\
0,21 * \\
0,35 * * * \\
0,26 *\end{array}$ & $\begin{array}{l}5,93^{* *} \\
3,54^{*} \\
1,87 \\
7,54^{* *} \\
2,72\end{array}$ \\
\hline $\begin{array}{l}\text { Activités } \\
\text { fonctionnelles } \\
\text { - Administration } \\
\text { - Relations publiques } \\
\text { - Innovation } \\
\text { - Régulation }\end{array}$ & $\begin{array}{r}28,90 \\
16,49 \\
3,38 \\
3,55 \\
5,48\end{array}$ & $\begin{array}{r}16,40 \\
13,57 \\
3,36 \\
4,90 \\
4,94\end{array}$ & $\begin{array}{r}35,62 \\
14,17 \\
6,93 \\
5,52 \\
8,99\end{array}$ & $\begin{array}{r}17,68 \\
12,63 \\
5,20 \\
3,46 \\
9,52\end{array}$ & $\begin{array}{r}46,28 \\
13,53 \\
9,70 \\
10,85 \\
12,20\end{array}$ & $\begin{array}{r}14,29 \\
9,67 \\
6,49 \\
10,05 \\
7,98\end{array}$ & $\begin{array}{l}0,30 * * \\
-0,04 \\
0,31 * * \\
0,30 * * \\
0,29 * *\end{array}$ & $\begin{array}{l}3,07 \\
0,19 \\
4,11^{*} \\
4,58^{*} \\
1,8\end{array}$ \\
\hline $\begin{array}{l}\text { Activités } \\
\text { opérationnelles } \\
\text { - Ventes } \\
\text { - Approvisionnement } \\
\text { - Maintenance } \\
\text { - Production }\end{array}$ & $\begin{array}{r}54,31 \\
25,46 \\
10,62 \\
1,92 \\
16,30\end{array}$ & $\begin{array}{r}26,06 \\
23,59 \\
8,51 \\
2,30 \\
25,39\end{array}$ & $\begin{array}{r}38,91 \\
16,27 \\
9,22 \\
3,22 \\
10,20\end{array}$ & $\begin{array}{r}23,21 \\
16,02 \\
6,29 \\
7,55 \\
17,38\end{array}$ & $\begin{array}{r}14,25 \\
10,16 \\
3,81 \\
0,19 \\
0,10\end{array}$ & $\begin{array}{r}13,64 \\
10,91 \\
6,04 \\
0,63 \\
0,30\end{array}$ & $\begin{array}{l}-0,48 * * * \\
-0,23 * \\
-0,31 * * \\
-0,27^{*} \\
-0,31 * *\end{array}$ & $\begin{array}{l}9,27 * * * \\
2,23 \\
3,24 * \\
1,10 \\
2,39\end{array}$ \\
\hline
\end{tabular}

$* \mathrm{p}<0,05 \quad * * \mathrm{p}<0,01 \quad * * * \mathrm{p}<0,001$ 
liées aux opérations, soit la production et l'approvisionnement (à 0,01), la maintenance et la vente $(0,05)$. Par contre et à l'exception de l'administration, le temps affecté aux autres activités augmente significativement à 0,001 pour la direction; à 0,01 pour la planification, les relations publiques, l'innovation et la régulation; et à 0,05 pour les activités de contrôle et d'organisation. Les résultats du test $\mathrm{F}$ donnent des différences significatives prévalant entre les stades en ce qui concerne globalement les activités opérationnelles (à 0,001) et de gestion (à 0,01 ), et en particulier pour 5 des 12 activités, soit la direction (à 0,01 ) et 1'innovation, les relations publiques, la planification ainsi que l'approvisionnement (à 0,05).

Au-delà de l'importance relative du temps consacré aux activités fonctionnelles, il ressort que, par rapport aux stades, les dirigeants allouent de moins en moins de temps aux activités opérationnelles, lesquelles sont progressivement déléguées. Inversement, ils allouent de plus en plus de leur temps aux activités de gestion. Par contre, l'étendue de leur champ d'activité est comparable d'un stade à l'autre.

\subsection{Perception des problèmes internes}

Nous avons mesuré l'importance respective de 19 types de problèmes, que nous avons classés en 5 catégories : marketing, management, finances, production et ressources humaines (tableau 3 ).

L'observation des moyennes montre globalement qu'aux stades de démarrage et de rentabilisation, les problèmes liés au marketing sont perçus comme les plus importants alors que ceux liés aux ressources humaines sont les moins importants. Par contre, au stade de réussite, les problèmes relevant de la gestion sont perçus comme les plus importants et c'est l'inverse, dans le cas des problèmes liés aux finances. Par ailleurs, l'éventail et la nature des problèmes perçus comme étant plus qu'importants (moyenne supérieure à 4) varient d'un stade à l'autre. Au démarrage, il s'agit de maintenir le niveau des ventes, d'acquérir une clientèle suffisante, de rendre l'entreprise viable, d'atteindre le seuil de rentabilité, de constituer un fonds de roulement suffisant, de financer la croissance et de rester rentable. Au stade de rentabilisation, on en dénombre deux: maintenir le niveau des ventes et acquérir une clientèle suffisante. Au stade de réussite, ces problèmes consistent à rester rentable, contrôler les performances, stabiliser la qualité des produits/services et acquérir une clientèle suffisante. Enfin, l'identité des problèmes les plus importants varie relativement à trois des cinq fonctions. Au démarrage, il s'agit au niveau du marketing de maintenir le niveau des ventes et d'acquérir une clientèle suffisante; au niveau financier, d'atteindre le seuil de rentabilité et d'occuper la main-d'œuvre, au niveau des 
ressources humaines. Au stade de rentabilisation, il s'agit sur le plan marketing de maintenir le niveau des ventes, de constituer un fonds de roulement suffisant sur le plan financier et d'occuper la main-d'œuvre à plein temps au niveau des ressources humaines. Au stade de réussite, il s'agit d'acquérir une clientèle suffisante au niveau marketing, de financer la croissance au niveau financier et de recruter des cadres au niveau des ressources humaines.

Le test de Kendall montre que l'importance de 7 des 19 problèmes varie significativement par rapport aux stades. Globalement, la tendance est significative pour les problèmes liés aux finances (à 0,05 ), dont l'importance diminue, et à la production et aux ressources humaines $(a ̀ ~ 0,01)$ qui, par contre, augmentent en importance. Les résultats détaillés montrent que l'importance de trois problèmes diminue significativement $(\mathrm{à} 0,05)$ : atteindre le seuil de rentabilité, constituer un fonds de roulement suffisant et rendre l'entreprise viable. Par contre, celle de cinq problèmes augmente significativement: recruter des cadres (à 0,001$)$, stabiliser le niveau (à 0,001$)$ et la qualité (à 0,05$)$ des produits/ services et contrôler les performances internes (à 0,01 ).

Les résultats du test de Kruskal-Wallis révèlent globalement des différenciations significatives en ce qui a trait aux problèmes liés à la production (à 0,01$)$. Les résultats détaillés montrent que des différences significatives prévalent entre les stades pour trois problèmes: stabiliser le niveau de production/ services, recruter des cadres (à 0,001$)$ et contrôler les performances internes (à 0,01$)$. Il s'agit donc là de problèmes qui sont plutôt caractéristiques des entreprises au stade de réussite. Ils suggèrent que les problèmes liés au marketing et aux finances sont plus caractéristiques des entreprises au stade de démarrage, alors que ceux liés à la production, aux ressources humaines et à la gestion sont plutôt caractéristiques des entreprises plus avancées dans leur développement. L'accroissement de la taille des entreprises permet d'expliquer la nature des différences retrouvées. Ces résultats sont globalement cohérents avec ceux rapportés par Vozikis et Glueck (1978), Kazajian (1984) et Dodge et Robbins (1992).

\subsection{Réseaux de relations externes}

Bien que plusieurs auteurs aient soulevé l'importance des relations dans l'étude du phénomène entrepreneurial (Dollinger, 1985; Aldrich et Zimmer, 1986), peu ont analysé empiriquement leur évolution. Pour les besoins de cette recherche, nous avons retenu 13 catégories de contacts externes. Un premier traitement des données a permis de constater des différences significatives par rapport aux stades $(a ̀$ a 0,01$)$ et entre les stades (à 0,05$)$ du nombre de types de contact entretenus par les dirigeants (tableau 4). 
TABleau 3

Perception de l'importance des problèmes ${ }^{1}$

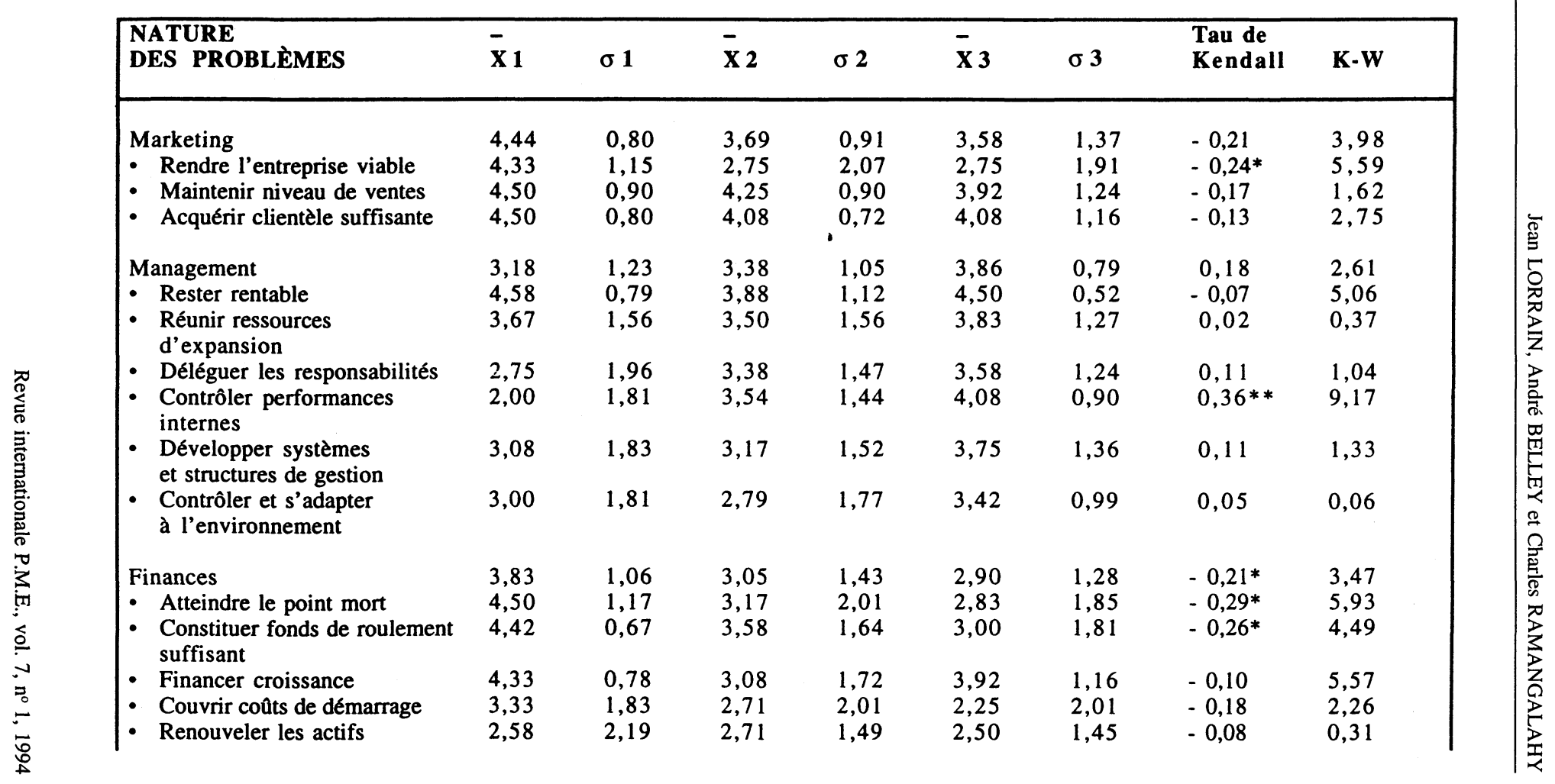




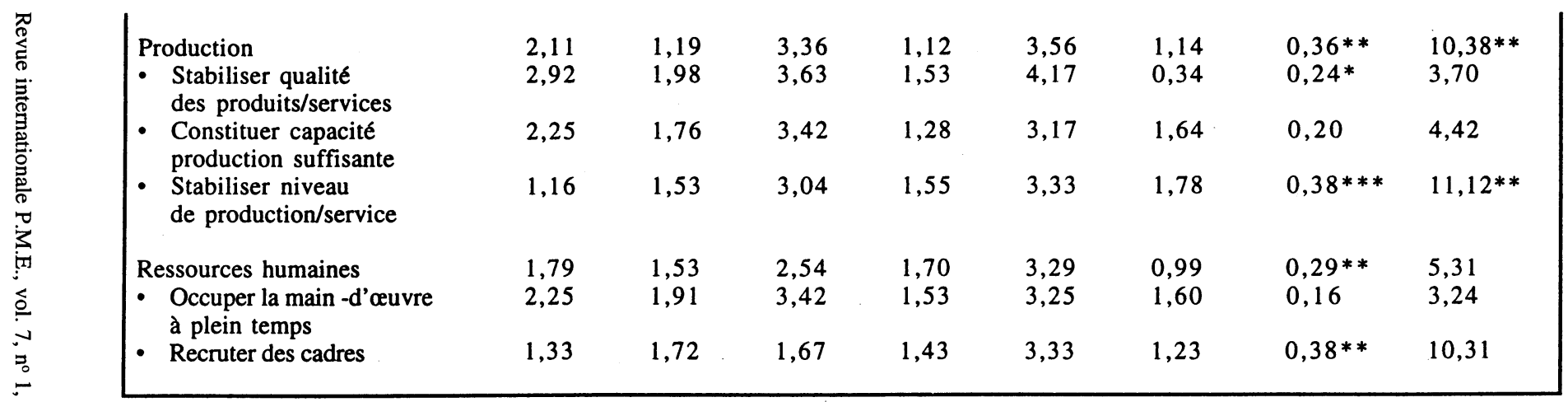

$* \mathrm{p}<0,05 ; \quad * * \mathrm{p}<0,01 \quad * * * \mathrm{p}<0,001$

1. Échelle allant de $1=$ Pas important à $5=$ Très important. 
TABlEAU 4

Nombre de catégories de contact par rapport aux stades

$\begin{array}{lc}\text { Nombre moyen de contacts par stade } & \\ \text { Démarrage } & 9,89 \\ \text { Rentabilisation } & 10,72 \\ \text { Réussite } & 12,17 \\ \text { Corrélation par rapport aux stades (Tau de Kendall) } & 0,39 * * \\ \text { Différenciation entre les stades (F-Oneway) } & 4,18^{*}\end{array}$

$* \mathrm{p}<0,05 \quad * * \mathrm{p}<0,01$

Ces premiers résultats indiquent donc une tendance significative à la diversification des relations par rapport aux stades. Nous avons analysé, par la suite, la perception de l'importance ainsi que la fréquence de chaque type de contact (tableau 5).

Quel que soit le stade, les dirigeants accordent le plus d'importance aux clients. L'inverse est observé concernant les syndicats, sauf au stade de réussite. Par ailleurs, on note un élargissement du noyau des contacts perçus comme importants. Au démarrage, ce noyau regroupe les clients, les fournisseurs et les comptables externes. Ces résultats confirment que le démarrage, suivant notre modèle théorique, est marqué par la prépondérance des activités opérationnelles. $\mathrm{Au}$ stade de rentabilisation, on retrouve en plus des clients, des fournisseurs et des institutions financières. Au stade de réussite s'y ajoutent les actionnaires/ associés ainsi que le conseil d'administration. On constate donc un mouvement de transfert des centres d'intérêt en matière de contacts externes d'interfaces à portée opérationnelle (clients et fournisseurs) vers des interfaces à portée stratégique (conseil d'administration, actionnaires/associés). Le test de Kendall donne des résultats significatifs quant à l'importance croissante accordée au conseil d'administration (à 0,001), aux syndicats et aux concurrents (à 0,01) et, enfin, aux consultants (à 0,05). L'importance des contacts avec les fournisseurs, les organismes de soutien aux PME et les comptables externes tend à diminuer sans être significative. Ces résultats peuvent être expliqués par le développement des structures d'organisation et des fonctions internes de l'entreprise. Le test Kruskall-Wallis révèle que les différences entre les stades ne sont significatives que dans le cas des relations avec les syndicats et le conseil d'administration (à 0,01 ). Il s'agit donc ici d'interfaces qui sont plus caractéristiques des entreprises au stade de réussite.

Les résultats relatifs à l'intensité des contacts, tout en étant cohérents avec les précédents, permettent de relever quelques nuances qui méritent d'être soulevées. On note une transposition des contacts les plus fréquents d'un noyau 
TABLEAU 5

Importance perçue ${ }^{1}$ et fréquence ${ }^{2}$ des contacts externes

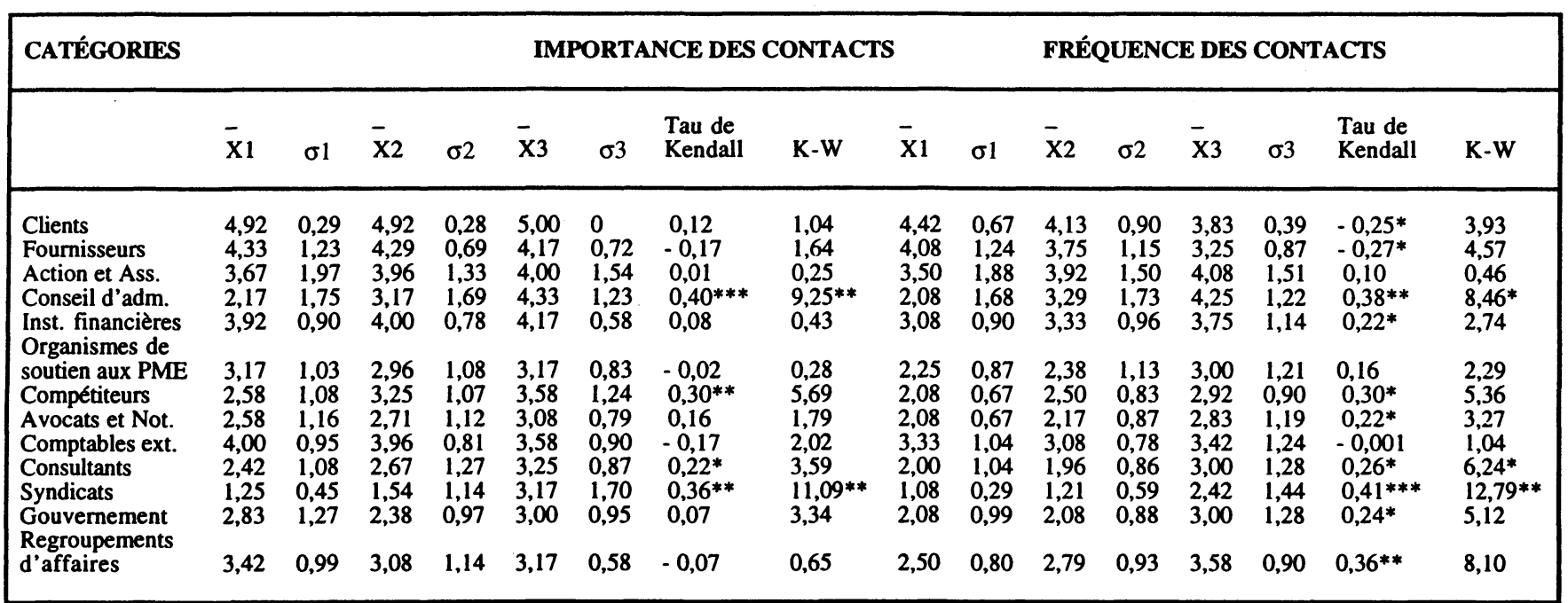

$* \mathrm{p}<0,05 \quad * * \mathrm{p}<0,01 \quad * * * \mathrm{p}<0,001$

1. Échelle allant de $1=$ Pas important à $5=$ Très important.

2. Échelle allant de $1=$ Jamais à $5=$ Toujours. 
d'interfaces à portée opérationnelle (clients et fournisseurs) vers un noyau d'utilité stratégique (conseil d'administration, actionnaires/associés). En effet, les résultas du test de Kendall montrent que les dirigeants entretiennent significativement de moins en moins de contacts avec les clients et les fournisseurs (à 0,05$)$. Cependant, le phénomène inverse est observé au sujet des syndicats (à 0,001 ), du conseil d'administration et des regroupements d'affaires (à 0,01 ), des compétiteurs, des consultants, du gouvernement et des avocats/notaires (à 0,05$)$. C'est ici que la tendance à la diversification des relations externes apparaît de façon claire. Les résultats du test de Kruskall-Wallis montrent que des différences entre les stades prévalent en ce qui concerne les syndicats $(0,01)$ et pour le conseil d'administration, les consultants et les regroupements d'affaires $(0,05)$.

Ces résultats sont cohérents avec la tendance observée dans l'évolution du profil d'activité des dirigeants. Au démarrage, les contacts à portée opérationnelle prédominent (clients et fournisseurs), mais leur importance et leur fréquence diminuent d'un stade à l'autre. Au stade de réussite prédominent progressivement les relations à portée managériale et stratégique (consultants, conseil d'administration, syndicats et regroupements d'affaires), sans que pour autant les relations avec les clients et les fournisseurs ne soient négligées.

\section{Conclusion}

Les résultats de notre recherche fournissent des indications relativement intéressantes autant sur l'évolution du comportement des dirigeants que sur l'orientation qu'il faudrait donner à l'avenir aux travaux de recherche dans ce domaine.

Le premier constat que nous faisons est qu'il y aurait peu d'intérêt à porter l'attention sur les différences éventuelles qui existent relativement à la nature des activités assumées par les dirigeants. Bien que nous ayons relevé une tendance à l'élargissement des activités assumées, il ressort que les dirigeants opèrent à l'intérieur d'un champ d'activité relativement homogène d'un stade à l'autre. Il est en particulier intéressant de noter l'absence de différenciation même au niveau des activités de gestion. Ces résultats montrent que le dirigeant d'une PME assume des activités de gestion et cela, dès le stade de démarrage. Il est d'autant plus pertinent de rappeler que les activités de gestion formaient $65 \%$ des variables que nous avons mesurées. Par ailleurs, cette recherche fournit un support non équivoque quant au fait que le dirigeant d'une PME assume des activités de planification stratégique, comme l'ont relevé d'autres chercheurs (Sexton et Van Auken, 1982). De telles activités prévalent dès le stade de démarrage. Toutefois, les résultats dont nous disposons ne nous permettent pas de nous prononcer ni sur la formalisation ni sur la complexité de ces pratiques. 
En second lieu, les résultats nous amènent à suggérer que l'attention devrait plutôt être portée sur l'implication et le temps que les dirigeants consacrent aux différentes activités. Les résultats confirment l'accroissement des pressions à la délégation en relation avec la croissance de l'entreprise, en particulier en ce qui concerne les activités opérationnelles, comme l'ont soutenu tous les auteurs qui traitent des modèles de développement (Greiner, 1972; Toulouse, 1982; Cooper, 1979). Par ailleurs, l'étude de l'utilisation que les dirigeants font de leur temps révèlent l'implication progressive des dirigeants dans la gestion (le pilotage) de leur entreprise, résultats appuyés par plusieurs chercheurs (Churchill et Lewis, 1983; Beckman et al. 1982; Broom et al. 1983). En effet, on observe une diminution significative du temps consacré aux opérations et, d'autre part, un accroissement significatif du temps consacré aux activités de gestion.

L'analyse de la perception que les dirigeants ont des problèmes organisationnels n'a pas permis d'identifier des différences significatives. On peut toutefois relever que les problèmes liés au marketing et aux finances sont plus caractéristiques des entreprises au stade de démarrage, alors que ceux liés à la production, aux ressources humaines et à la gestion sont plutôt caractéristiques des entreprises plus avancées dans leur développement. Ces résultats sont globalement cohérents avec ceux rapportés par Vozikis et Glueck (1978), Kazajian (1984) et Dodge et Robbins (1992).

L'étude de leurs réseaux de contacts a permis de relever une tendance significative à la diversification des relations externes des dirigeants. À cette tendance est par ailleurs associée une focalisation progressive des contacts vers des interfaces à portée stratégique, tels le conseil d'administration, le syndicat et les consultants externes.

Ces résultats, considérés globalement, permettent de soutenir que la capacité ou la possibilité pour le dirigeant de déléguer les opérations, de prendre en main progressivement le pilotage de son entreprise et de développer un réseau de contacts permettant de compléter les ressources internes sont des facteurs importants que le dirigeant, qui est intéressé par la croissance de son entreprise, doit considérer de près. Par ailleurs, la délégation des activités ainsi que le développement du réseau de contacts révèlent une concession accrue de la part du dirigeant concernant son autonomie et son indépendance dans la prise de décision et en matière de gestion.

Nous avons relevé un niveau généralement plutôt élevé des écart types. D'une part, cela renforce la pertinence des résultats que nous rapportons, dans la mesure où nous avons pu malgré tout identifier des différences significatives. D'autre part, ils nous suggèrent que les entreprises classées à un stade donné ne forment pas un groupe homogène. Ces données nous amènent à soulever, 
comme Vozikis et Mescon (1980), l'existence de microstades. En bref, notre modèle en est un de macro (ou méta) stades. C'est là, sans doute, l'une des plus importantes faiblesses, tant sur le plan théorique qu'opérationnel, de la modélisation de la croissance de la PME en stades.

Concernant l'opérationnalisation des modèles de développement, la pratique dominante a été de classer chaque entreprise étudiée à un stade donné. Cette approche suppose implicitement un processus continu, homogène ou synchrone du développement de l'ensemble des caractéristiques organisationnelles. Or, nos observations révèlent que tel n'est pas le cas. En effet, une entreprise peut se trouver à un stade donné du point de vue de ses composantes structurelles et à un autre stade lorsque considérée du point de vue de ses caractéristiques fonctionnelles. En d'autres termes, les diverses dimensions d'une entreprise n'évoluent pas de façon synchrone. Dans ce sens, le modèle opérationnel développé par Lorrain et Raymond (1992) rompt avec la «tradition ». À notre point de vue, leur approche pourrait mener à l'identification de microstades.

Quant aux activités des dirigeants, on oppose au niveau de la théorie deux écoles de pensée, soit l'école dite "classique» et celle dite de "l'activité quotidienne du dirigeant». Dans notre recherche, nous avons plutôt choisi de tirer profit de leurs apports respectifs. Une telle approche pourrait être critiquable, à priori, selon la perspective que l'on pourrait adopter pour définir ce que fait un dirigeant. Mais nous pensons fondamentalement qu'une telle approche semble plus appropriée au contexte de la PME.

Une autre limite de notre recherche tient au fait que nous avons restreint l'analyse des données à la vérification des différences par rapport et entre les stades. Nous n'avons pas procédé à des comparaisons intrastades, ce qui aurait permis de préciser davantage la pertinence des résultats obtenus. Ce type d'analyse devrait être encouragé, en particulier, pour tester la validité empirique des modèles de croissance.

Par ailleurs, nous avons analysé les comportements des dirigeants en relation seulement avec les facteurs organisationnels, que le concept de stade de développement permet d'appréhender globalement. Or, plusieurs auteurs ont relevé d'autres facteurs de contingence non moins importants, tels les profils du dirigeant et les caractéristiques de l'environnement de l'entreprise (Smith et Miner, 1983; Filley et Aldag, 1978; Dussault, 1987; Sandberg et Hofer, 1987). Aussi, est-ce à l'intérieur des limites relevées que les résultats doivent être appréciés.

En conclusion, au-delà des résultats que nous avons pu rapporter, nous réitérons les recommandations formulées par de plus en plus de chercheurs quant à la nécessité de réorienter les recherches sur l'étude de ce que fait l'entrepreneur pour expliquer sa réussite ou son échec. 


\section{Bibliographie}

ALDRICH, H. et C. ZIMMER (1985), «Entrepreneurship through social networks », dans D.L. Sexton et R.W. Smilor (éd.), Entrepreneurship, New York, Ballinger.

BASIRE, M. (1976), «La théorie des cinq niveaux », parties 2 et 3, Direction et Gestion, vol. 3, p. 11-12 et 4, p. 13-20.

BECKMAN, M.D., W.S. Good et R.G. WICKMAN (1982), "The life cycle of small business », Small Business Management - Concepts and Cases, Toronto, John Wiley \& Sons.

Broom, H.N., J.G. LONGENECKER et L.W. MOORE (1982), Small Business Management, $6^{e}$ édition, Cincinnati, Ohio, South Western Publishing Company.

CASTALDI, R.M. (1986), «An analysis of the work roles of CEO's of small firms », American Journal of Small Business, été 1986, vol. 11, n 1, p. 53-64.

Choran, I. (1969), The Manager of Small Company, Thèse non publiée, Université McGill, Montréal.

CHURCHILl, N.C. (1983), «Entrepreneurs and their enterprises : a stage model», dans J.A. Hornaday, J.A. Timmons et K.H. Vesper (éd.), Frontiers of Entrepreneurship Research, Wellesley, Ma, Babson College, Center for Entrepreneurial Studies.

ChurChill, N.C. et V.L. Lewis (1983), «Les cinq stades d'évolution d'une PME», Harvard l'Expansion, vol. 3, automne, p. 51-73.

COOPER, A. (1979), «Strategic management: new ventures and small business », dans D.E. Schendel et C.W. Hofer (éd.), Strategic Management: A New View of Management Policy and Planning, Boston, Little Brown and Company.

COOPER, A.C. et F.J.G. CASCON (1992), «Entrepreneurs, processes of founding, and new-firm performance», dans D.L. Schendel et J.D. Kasarda (éd.), The State of the Art of Entrepreneurship, Boston, PWS-Kent.

D'AMBoise, G. et M. Muldoney (1988), «Management theory for small business : attempts and requirements ", Academy of Management Review, vol. 13, $\mathrm{n}^{\circ} 2$, p. 226-240.

DeEks, J. (1976), "The owner-manager and his work», The Small Firm OwnerManager - Entrepreneurial Behavior and Management Practice, New York, Praeger Publishers.

DoDGE, R.H. et J.E. RoBBINS (1992), «An empirical investigation of the organizational life cycle model for small business development and survival », Journal of Small Business Management, vol. 30, $\mathrm{n}^{\circ}$ 1, p. 27-37.

DOLLINGER, M.J. (1985), «Environmental contacts and financial performance of small firm », Journal of Small Business Management, vol. 23, n 1, p. 24-30. 
DUSSAULT, L. (1987), Les comportements de gestion et les types d'entrepreneurs - cas des entreprises manufacturières en phase de démarrage, Rapport de recherche, Université du Québec à Trois-Rivières.

FILlEY, A.C. et R.J. AldAG (1980), «Organizational growth and types - lessons from small institutions », Research in Organizational Behavior, vol. 2, p. 279-320.

GABLE, M. et M. TOPOL (1987), «Planning practices of small-scale retailers », American Journal of Small Business, vol. 2, $\mathrm{n}^{\circ}$ 2, p. 19-32.

GARTNER, W.B. (1988), "Who is an entrepreneur? is the wrong question », American Journal of Small Business, vol. 12, $\mathrm{n}^{\circ} 4$, p. 11-32.

GREINER, L.E. (1972), «Evolution and revolution as organizations grow », Harvard Business Review, vol. 50, p. 37-46.

HALES, C.P. (1986), «What do managers do - a critical evidence», Journal of Management Studies, vol. 23, n 31, p. 88-115.

HEMPHILl, J.K. (1967), Dimensions of Executive Positions, Monographie ${ }^{\circ} 98$ publiée par le Bureau of Business Research, Ohio State University.

IBRAHIM, A.B. et J.R. GoDWIN (1986), «Perceived causes of success in small business », American Journal of Small Business, vol. 11, $\mathrm{n}^{\circ} 2$, p. 41-50,

KOTTER, J.P. (1982), «What effective managers really do », Harvard Business Review, novembre-décembre, vol. 60 , p. 157-167.

KAZAJIAN, R.K. (1984), «Operationalizing stage of growth - an empirical assessment of dominant problems », dans J.A. Hornaday, F. Tarpley, J.A. Timmons et K.H. Vesper (éd.), Frontiers of Entrepreneurship Research, Wellesley, Mass., Babson College.

KRoEger, C.R. (1974), "Managerial development in the small firm », California Management Review, vol. 17, $\mathrm{n}^{\circ}$ 1, p. 41-47.

LAFLAMME, M. (1981), Le management: approche systémique - théorie et cas, $3^{\mathrm{e}}$ édition, Chicoutimi, Gaëtan Morin Éditeur.

LORRAIN, J. et L. DUSSAULT (1988), «Relation between psychological characteristics, administrative behaviors and success of founder entrepreneurs at the start-up stage », dans B.A. Kirchhoff, W.A. Long, W.E. McMulan, K.H. Vesper et W.E. Wetzel Jr (éd.), Frontiers in Entrepreneurship Research, Wellesley, Mass., Babson College, p. 150-164.

LUTHANS, F. et D.L. LOCKWOOD (1984), «Toward an observation system for measuring leader behavior in natural settings » dans J.G. Hunt, D. Hosking, C. Schreisheim et R. Stewart (éd.), Leaders and Managers - International Perspectives on Managerial Behavior and Leadership, New York, Toronto, Pergamon Press.

MCEvoy, G.M (1984), « Small business personal practices », Journal of Small Business, vol. $22, \mathrm{n}^{\circ} 4$, p. $1-8$. 
MINER, J.B. et V.P. LUSCHINGER (1985), Introduction to Management, Colombus, Ohio, C.E. Merril Publishing Company .

MinTZBERG, H. (1984), Le manager au quotidien - les dix rôles du cadre, Traduction de l'américain, Paris, Éditions d'Organisation et Montréal, Agence d'Arc.

NAumes, W. (1978), The Entrepreneurial Manager in the Small Business, Don Mills, Ontario, Addison Wesley Publishing Company.

PHEYSEY, D.C. (1972), «Activities of middle managers - a training guide », The Journal of Management Studies, vol. 9, $\mathrm{n}^{\circ} 2$, p. 158-171.

PORTER, M.E. (1991), «Towards a dynamic theory of strategy », Strategic Management Journal, vol. 12, numéro spécial, hiver, p. 95-117.

QUINN, R.E. et K. CAMERON (1983), «Organizational life cycles and shifting criteria of effectiveness - some preliminary evidence », Management Science, vol. 29, ${ }^{\circ} 1$, p. 33-50.

RAYMOND, L. et J. LORRAIN (1992), «Computerisation as a factor in the development of young entrepreneurs », International Small Business Journal, vol. 11, $\mathrm{n}^{\circ} 1$, octobre-décembre, p. 23-34.

Robinson, R., J.A. PeArCe, G. Vozikis et T. MesCon (1984), «The relationship between stade of development and the small firm planning performance », Journal of Small Business Management, vol. 22, $\mathrm{n}^{\circ} 2$, p. 45-52.

SANDBERG, W.R. et C.W. HoFER (1987), «Improving new venture performance : the role of strategy, structure and the entrepreneur », Journal of Business Venturing, vol. 2, p. 5-28.

SAYLES, L.R. (1964), Managerial Behavior, New York, McGraw-Hill Company.

SCOTT, B.R. (1973), «The industrial state: old myths and new realities », Harvard Business Review, vol. 51, p. 133-148.

SeXton, D.L. et P.M. VAN AUKEN (1982), «Prevalence of strategic planning in the small business », Journal of Small Business Management, vol. 20, $n^{\circ} 3$, juillet, p. 20-26.

SMITH, K.G., T.R. MitChELl et C. SUMER (1985), « Top level management priorities in the different stages of organizational life cycle», Academy of Management Journal, vol. $28, n^{\circ} 4$, p. 799-820.

SMITH, N.R. et J.B. MINER (1983), «Type of entrepreneur, type of firm, and managerial motivation : implications for organizational life cycle theory », Strategic Management Journal, vol. 4, n 3, p. 325-340.

STANWORTH, M.J.K et J. CURRAN (1976), «Growth and the small firm - an alternative view », Journal of Management Studies, vol. 13, $\mathrm{n}^{\circ} 2$, p. 95-100.

Stegal, D.P., L.L. Steinmetz et J.B. KLINE (1976), Managing the Small Business, Homewood, Richard D. Irwin, chapitre 10, p. 149-155. 
STEINMETZ, L.L. (1969), «Critical stages of small business growth », Business Horizons, vol. $12, \mathrm{n}^{\circ} 1$, p. 29-36.

STEVENSON, H.H. et J.C. JARILlo (1990), «A paradigm of entrepreneurship: entrepreneurial management », Strategic Management Review, vol. 11, numéro spécial, p. 17-27.

STEWART, R. (1986), The Reality of Management, $2^{\mathrm{e}}$ éd., London et Sidney, Éditions Pan Books.

Susbauer, D.H. (1979), Commentaires sur l'article de Cooper, dans D.E. Schendel et C.W. Hofer (éd.), Strategic Management: A New View of Management Policy and Planning, Little Brown and Company.

THAIN, D.H. (1969), «Stages of corporate development », Business Quarterly, vol. 34, $\mathrm{n}^{\circ} 4$, p. 32-46.

TORNOW, W.W. et P.R. PINTO (1976), «The development of a managerial job taxonomy - a system for describing, classifying and evaluating executive positions », Journal of Applied Psychology, vol. 61, $\mathrm{n}^{\circ} 4$, p. 410-418.

Toulouse, J.M. (1982), «Stades de développement de l'entreprise et les défis de l'entrepreneur », Revue Commerce, vol. 16, p. 124-134.

VAN AUKEN, P.M. et R.D. IRELAND (1978), «An historical review of management philosophy », Academy of Management Review, J.C. Susbauer (éd.), Cleveland State University, p. 7-11.

VozIKIS, G. et W. GLUECK (1978), «Small business and stages of development », Academy of Management Proceedings, 38th Annual Meeting, édité par J.C. Susbauer, Cleveland State University, p. 373-376.

VozIKIS, G. et W. GLUECK (1980), «Small business problems and stages of development », Academy of Management Proceeding, 40th Annual Meeting, édité par R. Huseman, Jeffrey, University of Georgia, p. 373-377.

VOZIKIS, G. et T. MESCON (1985), «Small exporters and stages of development: an empirical study », American Journal of Small Business, vol. 10, $n^{\circ} 1$, p. 49-69. 